\title{
Dell'imperfetto commemorativo, funebre, funereo o funesto
}

\author{
Marco MAZZOLENI \\ SITLeC e SSLMIT, Università di Bologna \\ Polo Scientifico-Didattico di Forli*
}

Recibido: $16 / 07 / 2010$

Aceptado: 20/12/2010

\section{RIASSUNTO}

In italiano c'è un uso dell'indicativo imperfetto che sembra suggerire che lo stato di cose presentato dal mittente non è semplicemente passato, cioè avvenuto in un momento che precede cronologicamente quello dell'enunciazione, ma risulta anche irrevocabilmente finito, concluso, terminato - nel presente non si dà più: se di qualcuno si dice che era proprio un bravo ragazzo il destinatario tende spontaneamente a pensare che la persona in questione è scomparsa, oppure che il suo comportamento abituale è decisamente peggiorato. In questo articolo si tenta di illustrare brevemente quest'uso dell'imperfetto in alcuni generi testuali e discorsivi dell'italiano moderno e contemporaneo, affiancandolo all'uso del perfetto semplice di essere reso indimenticabile dal manzoniano «Ei fu» ma comunque anche tipico della lingua burocratico-amministrativa, tra l'altro ironicamente evocata da Pirandello con $\mathrm{Il}$ fu Mattia Pascal.

Parole chiave: Indicativo imperfetto, perfetto semplice, testi/discorsi commemorativi, aspetto.

\section{On the 'commemorative' use of the Italian Indicativo Imperfetto}

\begin{abstract}
There is a particular use of the Italian imperfect indicative that appears to suggest that the state of affairs presented by the speaker is not simply situated in the past - i.e. happened at a time chronologically previous to the utterance - but also something that is now finally and

* Corso della Repubblica 136, I-47121 Forlì FC Italia; marco.mazzoleni@unibo.it. Per l'aiuto ricevuto durante la stesura di questo articolo voglio ringraziare Derek Boothman, Andrea Cristiani, Francesco Giardinazzo, Yvonne Grimaldi, Francesca La Forgia, Michela Massa, Maria Enriqueta Pérez Vázquez, Giampiero Pizzol e Sam Whitsitt, mentre la responsabilità delle imperfezioni e degli errori residui va al solito ascritta all'autore.
\end{abstract}


irrevocably closed and finished, i.e. a state of affairs that no longer exists. If in Italian one says «s/he was (era) a fine person», this automatically suggests that the person spoken of has passed on and is no longer with us, or alternatively that his/her habitual behaviour has taken a decisive turn for the worse. This research intends to illustrate briefly this use of the imperfect in a number of discourse and textual genres in modern and contemporary Italian, alongside with the use of the simple past (perfetto semplice) of essere, typified in Manzoni's unforgettable «Ei fu» («he has passed» in some translations of his poem The Fifth of May), a use now typical of the bureaucratic language referred to ironically in the title of Pirandello's Il fu Mattia Pascal (The late Mattia Pascal).

Key words: Imperfect indicative, Simple past, Commemorative Texts / Speeches, Aspect.

This was my son.

Notice how I said «was»?

That's because he's dead. Relegated to the past tense. Went from an is to a was before he had his breakfast.

\section{IL PASSATO NON $C^{\prime} \grave{E}$ PIÙ...}

Come credo risulti evidente dal frammento citato in ex ergo, tratto da una sequenza del film Lucky Number Slevin di Paul McGuigan (USA 2006, con fra gli altri Ben Kingsley, Lucy Liu e Bruce Willis) in cui il Boss, personaggio interpretato da Morgan Freeman, sta mostrando al suo interlocutore una fotografia del figlio morto, a(lmeno alcune) volte l'uso di un Tempo ${ }^{1}$ passato non colloca semplicemente un'azione, un evento, una situazione - cioè quanto in modo più astratto possiamo chiamare uno «stato di cose» - in un momento che precede cronologicamente il momento dell'enunciazione, ma sembra suggerire (o per lo meno consente al destinatario di inferire) che quello stato di cose nel presente non si dà più, si è concluso, è finito, risulta irrimediabilmente terminato: se dico «This was my son» indicandone la fotografia implico che mio figlio non è più...2.

1 Per evitare possibili equivoci, distinguo come d'uso il Tempo verbale (una categoria linguistica di carattere principalmente morfologico, con le sue componenti semantico-pragmatiche) dal tempo crono- o biologico, legato all'entropia - mentre non ritengo necessaria una ulteriore distinzione terminologica per l'uso della parola in senso 'climatico', che compare ad es. nel titolo della nota trasmissione televisiva Che tempo che fa, condotta da Fabio Fazio ed in onda nella fascia pre-serale su RAI 3 nel fine settimana.

2 Una dichiarazione come «(Lui/Lei) Non è più mio/a figlio/a» suona invece come un disconoscimento, una risoluzione del rapporto sociale di parentela - ma non certo di quello naturale, biologico! -, che a quanto ne so può avvenire però solo nella direzione da genitori a figli: non mi pare infatti che (per lo meno nella legislazione corrente) un/a figlio/a possa disconoscere sua madre o suo padre, oppure che nonne/i o zie/zii possano disconoscere dei/lle nipoti o viceversa, o ancora che ci si possa disconoscere tra fratelli/sorelle o cugini/e. Più in particolare, un disconoscimento mi sembra un atto di rescissione di un rapporto di paternità: come un padre può riconoscere ufficialmente la sua progenie - una madre non può farlo, perché come recita l'adagio latino (ma attenzione agli scambi di neonati...) mater sempre certa -, così può disconoscerla. 
Si potrebbe pensare che questo effetto discorsivo dipenda non tanto o non solo dal Tempo verbale, quanto piuttosto (anche) dalla presenza del verbo essere, che fin dai tempi della filosofia greca - matrice di tanta parte della cultura occidentale cui noi apparteniam - è caratterizzato da forti connotazioni esistenziali, o ancora dal fatto che dal punto di vista semantico-lessicale il predicato sia costituito da un kinterm, cioè un nome relazionale che instaura tra i (referenti dei) suoi due argomenti un rapporto di parentela ${ }^{3}$. Una relazione parentale naturale, non acquisita, non viene infatti meno se non con l'estinzione di uno dei due parenti, o meglio: un kinterm non è più predicabile del suo argomento-soggetto solo quando il relativo referente ha cessato di esistere, perché il rapporto di parentela in quanto tale sussiste ancora anche se risulta predicabile soltanto con il termine converso - se mio figlio è morto, non per questo si è conclusa la mia paternità ed io non sono più padre ${ }^{4} .$. Ma questo effetto di inevitabilmente definitiva collocazione nel passato di uno stato di cose non accade soltanto con il verbo essere combinato con un termine di parentela: dai versi seguenti, che costituiscono la terza strofa della nota poesia Funeral Blues del grande poeta americano Wystan Hugh Auden (pubblicata in Another Time, London, Faber \& Faber, 1940: 91), si capisce non solo che il defunto commemorato e compianto non rappresenta purtroppo più i punti di riferimento dell'autore implicito, ma anche che questi non pensa più che l'amore possa durare per sempre e che quindi - sia pur tristemente - non si sbaglia più.

He was my North, my South, my East, and West, My working week and my Sunday rest,

My noon, my midnight, my talk, my song;

I thought that love would last forever: I was wrong.

\section{2. «IL FU»}

I due casi inglesi che ho discusso finora provengono evidentemente da testi o discorsi dal tono elegiaco e di carattere in qualche modo commemorativo. Nella tradizione culturale italiana l'elogio funebre probabilmente più noto è $\mathrm{Il}$ cinque maggio di Alessandro Manzoni ${ }^{5}$, con il suo potente ed icastico incipit «Ei fu» al

3 Sull'organizzazione interna del campo semantico-lessicale dei termini di parentela e sui loro varî rapporti di senso cfr. ad es. Mazzoleni (1997: § 2.1) e la relativa bibliografia.

${ }^{4}$ Invece le relazioni parentali acquisite possono concludersi anche senza la morte dei congiunti, come accade ad es. con il divorzio: così si può dire «Lei era mia moglie / Lui era mio marito» anche quando la persona cui ci si riferisce è ancora in vita, pur avendo a disposizione l'alternativa non equivoca «Lei/Lui è la mia ex-moglie/il mio ex-marito». Discorso diverso va fatto per l'annullamento, procedura giuridica prevista dalla chiesa cattolica, perché la sentenza emessa dal tribunale ecclesiastico della Sacra Rota comporta che il matrimonio in questione non c'è mai stato, e quindi in un caso del genere non avrebbe senso parlare di exconiugi.

5 Scritto in morte di Napoleone Bonaparte fra il 17 ed 19 luglio 1821, lo si può leggere ad es. nella sezione Inni sacri e Odi delle Opere di Alessandro Manzoni curate da Riccardo Bacchelli, Milano-Napoli, Ricciardi, 1973. 
perfetto semplice (nella tradizione grammaticale scolastica italiana «passato remoto»), forma verbale che domina l'intera ode, innesca lo stesso 'effetto collaterale' visto prima con il simple past inglese - dire di qualcuno che «fu» implica che quel qualcuno non è più -, e che con la visualizzazione aspettuale 'compatta', conclusa e compiuta offerta al lettore appare perfetta per il genere cui il testo appartiene.

D'altronde la terza persona singolare del perfetto semplice di essere è connessa ai defunti da una storia piuttosto antica all'interno della lingua italiana, in particolare nelle sue varietà burocratico-amministrative e specialmente per le identificazioni anagrafiche: non è certo questo il luogo per approfondire tale storia, ma ne voglio fornire sia pure in modo aneddotico almeno alcuni frammenti, tratti da tre diversi tagli sincronici.

\subsection{Nel XVI secolo}

I primi esempî che voglio presentare risalgono al 1584, e provengono da documenti dell'Archivio di Stato di Genova (sala Senarega, filza $n^{\circ} 526$ ) resi disponibili da Guido Ferretti nel suo articolo Un anomalo incidente di caccia nel 1584 (cfr. http://www.valdaveto.net/documento_192.html): negli atti dell'inchiesta della Corte di Roccatagliata sull'evento, accaduto vicino a Cicagna in località Prato della Piana, si può leggere innanzitutto che:

Benedetto Ratto Console di Campo de Zasco Podest.ria di Rocc.ta, denuncia a me Fran.co Poggio Podestà in qualmente hieri sera che potea essere hore 19 in circa fu morto una donna nominata Lucia moglie di Giacomo Levorone in loco detto Prato della Piana in la detta Consol.ia Giur.ne di Rocc.ta: E per quello che si dice pubblicamente esser statta morta di una archibuggiata da Tognino Porcella del fu Gioanni del Ponte di Cicagna della Cànova. [1584 adì 8 giugno - in la Corte di Rocc.ta]

L'autore della «archibuggiata» viene identificato non soltanto tramite il suo nome ma anche con riferimento al suo defunto padre, il « $u$ Gioanni del Ponte di Cicagna della Cànova»; e come si vede dalla citazione seguente medesimo trattamento è riservato ai tre testimoni coinvolti nella procedura ufficiale di riconoscimento della salma ( Giacomollo Cazazza del fu Benedetto e Sentino Cazazza del fu Pasquale et Battino Bacigalupo del fu Gieronimo»):

Visto e visitato per me sudetto Podestà in presentia di Giovanni Schiappacazze messo pubblico della corte e degli infrascritti testimonij. Il cadavero della fu Lucia moglie di Giacomo Levorone e figlia di Lazarino Levorone postrata in terra morta, statta ferita di una archibuggiata botta di quadrello per quello [che] si può comprendere in la ponta della mascella dritta, di età d'anni 26 in circa secondo che mostra il suo aspetto, vestita di bianco, e quanto è statta morta per quello dicono li soi di casa era vestita di una roba di raxa verde. In presentia di Giacomollo 
Cazazza del fu Benedetto e Sentino Cazazza del fu Pasquale et Battino Bacigalupo del fu Gieronimo che sopra ciò si sono essaminatti per me sudetto Podestà, datoli prima giuramento, li quali attestano che il detto cadavero era di detta Lucia che così si chiamava in vitta. [adì q. detto - In casa di Lazarino Levorone loco detto la Cànova al ponte di Cicagna]

A questo punto anche la stessa vittima comincia a comparire con questa 'marca da defunta' - «[...] la fu Lucia moglie di Giacomo Levorone e figlia di Lazarino Levorone» -, anche se come si può vedere dal prossimo frammento citato la sua catena anaforica (dal forte sapore burocratico-amministrativo!) può risultare anche costituita da sintagmi complessi del tipo di «detta Lucia che così si chiamava in vitta», o ancora «detta Lucia morta» e «detta Lucia mia figliola», quest'ultimo ovviamente dalla bocca del padre, che a sua volta viene di nuovo identificato anche con riferimento al suo defunto padre - ovvero al nonno della deceduta: «Lazarino Levorone $f u$ Lodisio della Cànova del ponte di Cicagna».

Lazarino Levorone $f u$ Lodisio della Cànova del ponte di Cicagna padre della detta Lucia morta citato per informazione in detta causa dattoli giuramento di dire la verità, interrogato sopra detta causa, per suo giuram.to testificando dice. Il giorno di hieri che potteva essere da hore 19 in circa essendo nel presente loco qui accertato in terra in compagnia della detta Lucia mia figliola [...]. [adì detto in loco dove si chiama il Prato della Piana Consolaria di Campo di Zasco Podesteria di Roccatagliata, nel loco proprio dove è stato morto la detta Lucia]

Manca in quest'ultimo caso l'articolo definito, in una variante che risulta comune nella formulazioni anagrafiche e che viene utilizzata anche dallo stesso autore dell'articolo in un suo commento di raccordo tra i diversi documenti d'archivio presentati: «Durante l'inchiesta il Podestà di Roccatagliata sentì, inoltre, le testimonianze di Giacobo Cazazza $f u$ Pietro di Ponte di Cicagna, di Don Francesco Garbarino, arciprete della chiesa di S. Giov. Battista del Ponte di Cicagna e di Gregorio Porcella anch'esso abitante a Ponte di Cicagna» (Ferretti, cit. supra ad inizio paragrafo [http://www.valdaveto.net/documento_192.html]).

\subsection{Nel XVIII secolo}

Un secondo esempio che illustra quest'uso della terza persona singolare del perfetto semplice di essere nell'identificazione di defunti proviene da un libro pubblicato a Venezia nel 1752, dedicato ai funerali bolognesi «del fu Sua Eccellenza F. Francesco-Saverio Conte Marulli», che compare in questo modo nel lungo e complesso titolo del volume di cui di séguito riproduco con grafia normalizzata il frontespizio, e che nella Relazione che inizia il testo - riportata sotto - viene ripreso con la formula identificativa che ormai abbiamo visto più volte (il facsimile digitalizzato dell'originale si può trovare al seguente indirizzo: http:/www.archive.org/details/ funeralidelfusua00inve): 


\author{
FUNERALI \\ DEL FU SUA ECCELLENZA \\ F. FRANCESCO-SAVERIO \\ CONTE MARULLI \\ Gran Priore di Venezia, Commendatore della Baliale Com- \\ menda di S. Maria del Tempio di Bologna, e Cavaliero di \\ Gran Croce per l'Ordine Gerosolimitano di Malta; \\ Maresciallo di Campo, Consigliero di Guerra, Colonello di un \\ Regimento di Fanteria, e Cavaliere della Chiave d'Oro \\ delle Loro Maestà Imperiale e Reale \&c.\&c. \\ FATTESI CELEBRARE \\ DA SUA ECCELLENZA IL SIG. DON \\ GIACOMO CONTE MARULLI \\ SUO PRONIPOTE \\ Nella detta Chiesa di Sua Residenza di Santa Maria \\ del Tempio di Bologna. \\ IN VENEZIA, MDCCLII.
}

CON LICENZA DE' SUPERIORI.

Perchè irreparabili, e grandi si compiangono sempre le perdite degli Uomini illustri; fu perciò a ragione quella universalmente compianta che si ebbe la notte precedente il giorno venti di Agosto dell'Anno, 1751 del fu Eccellentissimo Signor Maresciallo Fra Francesco Conte Marulli, perchè di ragguardevole Cavaliere rinomatissimo per le morali egregie virtù sue del pari, che per le gloriose sue guerriere azioni.

\title{
2.3. Nel XIX secolo
}

Una terza serie di casi proviene da un genere testuale che ritengo più 'confortante' dei precedenti, visto che si tratta di pubblicazioni di matrimonio: utilizzo come esempî alcune di quelle messe all'albo del Comune di Montemiletto (Benevento) tra il gennaio ed il dicembre del 1811 - disponibili in rete all'indirizzo http://tlngenealogy.blogspot.com/2010/01/1811-montemiletto-marriage-banns. html. In questi Atti di Stato Civile i futuri sposi vengono identificati anagraficamente non solo in base al loro nome e cognome ed eventuale professione ma anche in quanto figli dei rispettivi genitori, uno dei quali a volte è morto e compare quindi come «il/la fu» (quando sono mancati entrambi i genitori la formula è «figlio/a delli defunti...»); in qualche caso poi uno/a dei futuri sposi è anche

${ }^{6}$ L'identificazione di una persona tramite i suoi genitori è peraltro una modalità assai frequente anche nelle culture popolari tradizionali: senza bisogno di ricorrere ad esempî esotici provenienti da ricerche etnoantropologiche su lontane popolazioni primitive, basti pensare che a tutt'oggi in romagnolo la maniera più comune (ed affatto offensiva!) per chiedere a qualcuno chi è consiste nel domandargli «Ad chi sit e' fiol te?» [Di chi sei il figlio tu?] - e la frase può risultare a volte persino priva del kinterm e suonare quindi «Ad chi sit te?» [Di chi sei tu?]. 
vedovo/a, ed anche in questo caso al coniuge defunto si fa riferimento con «il/la fu»:

Sposo: Biaggio di Iovanna, 31, campagnuolo, figlio di Giovanni di Iovanna e della fu Giuseppa di Virgilio

Sposa: Rosa Sacco, 20, figlia del fu Carmine Sacco ed Angela Maria Brogna

Sposo: Fiorentino Sacco, 22, bracciale, dom. Montaperto, figlio del fu Angelo Sacco ed Angiola Maria (Angiolillo)

Sposa: Maria Emanuella Anfieri, 28, contadina, figlia di Gaetano Anfieri e della fu Marianna Fiorentino

Sposo: Francesco Capone, 32, campagnuolo, figlio di Vincenzo Capone e Gaetana Sacco

Sposa: Rosa Selvitella, 35, vedova del fu Ciriaco di Fronzo, figlia delli defunti Nicola Selvitella ed Angiola Maria Carpenito

Sposo: Romualdo Ambrosio, 40, bracciale, di Pietradefusi, vedovo della fu Saveria Ciriello di Montemiletto, figlio delli defunti Palerio Ambrosio e Leonilla de Nisco

Sposa: Teresa Corbo, 46, contadina, figlia delli defunti Iganzio [Ignazio?] Corbo ed Elisabetta Petrillo

Infine voglio ricordare come questa modalità di identificazione dei defunti sia stata anche sfruttata con una certa ironia da Luigi Pirandello ne Il fu Mattia Pascal, il cui protagonista (dato per suicida da un quotidiano, e considerato anagraficamente morto in base al riconoscimento ufficiale del cadavere da parte di moglie e suocera) è in realtà ancora vivo e vegeto, e coglie al volo l'occasione di iniziare una nuova vita con il nome di Adriano Meis: in questo caso è appunto la sua identità 'anagrafica' ad essere defunta e non quella personale, e tale divaricazione fra le molteplici possibili componenti identitarie di un individuo è tema pirandelliano quant'altri mai...

\section{L'IMPERFETTO COMMEMORATIVO}

Passando però dall' «Ei fu» della succitata ode manzoniana e dal(l')«(il/la) fu» della lingua della tradizione burocratico-amministrativa all'italiano contemporaneo, si scopre che la forma verbale più tipica - anche se non esclusiva - dei testi e dei discorsi funebri e commemorativi è l'indicativo imperfetto, testimoniato in diverse varietà sia nell'orale che nello scritto.

\subsection{Nell'italiano del doppiaggio e della traduzione}

Innanzitutto lo troviamo nel doppiaggio italiano della battuta del film citata in ex ergo, dove la forma verbale occorre con un valore nel quale alla componente temporale di passato deittico si affianca un senso di irrimediabile e nostalgica compiu- 
tezza che pare opporsi alla valenza aspettualmente imperfettiva tipica del suo significato di base ${ }^{7}$ :

Questo era mio figlio.

Avrai notato che ho detto «era».

Ora è morto... Ammazzato. Relegato all'imperfetto dell'essere.

Spedito da un è a un era prima di fare colazione.

(Paul McGuigan, Slevin - Patto criminale, USA 2006)

La soluzione traduttiva adottata non è per nulla letterale, ed appare evidentemente motivata dal fatto che in italiano una resa del simple past dell'originale inglese con il perfetto semplice o composto («Questo $f u$ / è stato mio figlio») sarebbe stata del tutto inappropriata: la scelta della forma verbale ha poi chiaramente obbligato la conseguente modifica di «Relegated to the past tense» in «Relegato all' imperfetto»; ma qui scatta secondo me il colpo di genio della versione italiana, perché invece di essere completato con un poco comprensibile o banale «di essere / del verbo essere» l'enunciato prosegue con «dell'essere», dove l'articolo innesca il senso dell'uso sostantivato dell'infinito ${ }^{8}$.

Troviamo di nuovo l'imperfetto - ed anche con una maggiore ed intensa frequenza rispetto ai tre simple pasts dell'originale - nella traduzione italiana (in Un altro tempo, edizione con testo a fronte a cura di Nicola Gardini, Milano, Adelphi [Biblioteca Adelphi 345], 1997) dei versi di W. H. Auden citati alla fine del §1:

Lui era il mio Nord, era il mio Sud, era l'Oriente e l'Occidente, I miei giorni di lavoro, i miei giorni di festa,

Era il mezzodì, la mia mezzanotte, la mia musica, le mie parole;

Credevo che l'amore potesse durare per sempre: era un'illusione.

Tra l'altro la quartina compare anche nel film di Mike Newell Four Weddings and a Funeral (GB, 1994), con fra gli altri Hugh Grant, Andie MacDowell e Kristin Scott Thomas, all'interno dell'orazione funebre pronunciata da Matthew (interpretato da John [David] Hannah) in memoria di Gareth (impersonato da Simon [Phillip

\footnotetext{
7 In Mazzoleni (2009) ho però tentato di mostrare lo 'sbiadimento' delle caratteristiche temporali, aspettuali e modali dell'indicativo imperfetto testimoniato dai suoi diversi possibili usi nell'italiano contemporaneo (nella citazione sopra riportata si noti tra l'altro anche l'uso epistemico del futuro composto - tradizionalmente «anteriore»-avrai notato).

${ }^{8}$ Numerosi sono gli esempî dell'eccellenza della tradizione del doppiaggio italiano (per quanto nato dalla xenofobia anche linguistica del ventennio fascista, e dalla qualità ultimamente purtroppo un po' in declino): fra tutti basti ricordare la magnifica resa «Lupo ulula? - Lupo ululà, castello ululì» di Frankestein Junior, soluzione che supera di gran lunga il già pur splendido scambio originale «Werewolf? - Where wolf? There wolf and there castle» del parodico Young Frankenstein di Mel Brooks (USA 1974), con il fraintendimento tra Frederick (interpretato da Gene Wilder), il nipote dell'ormai defunto barone, ed Igor (impersonato dal grande Marty Feldman), antico servitore del nonno Viktor, dalla gobba - sia pur denegata: «Gobba? Quale gobba?» - a posizionamento variabile tra la scapola destra e quella sinistra; tra l'altro Igor, sentendo la pronuncia americanizzata del cognome di famiglia («Frankenstin») pretesa da Frederick, decide immediatamente che il suo nome dovrà essere pronunciato «Eye-gor» - con una molto probabile allusione allo strabismo divergente ed all'esoftalmia caratteristici dell'attore che lo interpreta...
} 
Hugh] Callow); nella versione italiana (Quattro matrimoni e un funerale) il testo utilizzato nel doppiaggio della lirica di Auden corrisponde sostanzialmente alla traduzione sopracitata, ma nella parte precedente della commemorazione si trovano anche altri imperfetti del tipo che stiamo discutendo - «Diceva che» $\Rightarrow$ 'ora non dice più', la sua «gioia $[. .$.$] esplodeva» \Rightarrow$ 'ora non esplode più', e «quando si ubriacava» $=>$ 'ora non si ubriaca più':

Gareth ha sempre preferito i funerali ai matrimoni. Diceva che... per lui era più facile entusiasmarsi per una cerimonia di cui prima o poi sarebbe stato il protagonista. [...]. Molti di noi rimpiangeranno la sua incredibile e straordinaria gioia di vivere: una gioia... una gioia che esplodeva soprattutto quando si $u b r i a-$ cava.

Gli esempi che ho presentato finora di quest'uso dell'imperfetto in frammenti di testi e discorsi di carattere commemorativo provengono dall'italiano del doppiaggio cinematografico e della traduzione, e quindi si potrebbe anche pensare che il fenomeno dipenda da questo tipo di procedure di trasposizione linguistica, che tra l'altro nello specifico avevano in entrambi i casi l'inglese come lingua di partenza. L'organizzazione del sistema tempo-aspettuale delle forme verbali dell'inglese è però notoriamente diversa rispetto a quella dell'italiano, e questa differenza rappresenta anche una seria difficoltà nell'insegnamento sia dell'inglese ad apprendenti italofoni che dell'italiano ad apprendenti anglofoni: non c'è infatti una corrispondenza biunivoca tra le forme verbali delle due lingue, come si può anche vedere confrontando ad es. l'originale ed una delle traduzioni italiane di parte del discorso funebre di Antonio sul cadavere di Giulio Cesare, dalla $2^{\mathrm{a}}$ scena del III Atto del noto dramma shakespeariano, dove i diversi casi di simple past dell'inglese sono resi in italiano a volte con l'imperfetto, a volte con il perfetto semplice ed a volte con quello composto (nei due testi le forme verbali parallele sono evidenziate in corsivo), ed addirittura il famosissimo frammento "Cæsar / He was ambitious» viene tradotto una prima volta con «era ambizioso» e le altre con «fu ambizioso». ${ }^{9}$

[...] The noble Brutus

Hath told you Cæsar was ambitious:

If it were so, it was a grievous fault,

And grievously hath Cæsar answer'd it.

$[\ldots]$

He was my friend, faithful and just to me:

But Brutus says he was ambitious;

And Brutus is an honourable man.

He hath brought many captives home to

Rome,
Il nobile Bruto vi ha detto che Cesare era ambizioso:

se cosìera, $f u$ un ben grave difetto: e

gravemente Cesare ne ha pagato il fio.

$[\ldots]$

Eglifu mio amico, fedele e giusto verso di me:

ma Bruto dice che $f u$ ambizioso; e Bruto è uomo d'onore.

Molti prigionieri egli ha riportato a Roma,

${ }^{9}$ Cfr. per l'originale The Works of William Shakespeare, a cura di William George Clark e William Aldis Wright, Cambridge / London, MacMillan and Co., 1864, e per la traduzione Shakespeare. Tutte le opere, a cura di Mario Praz, Firenze, Sansoni, 1964, 1970', p. 604. 
Whose ransoms did the general coffersfill: Did this in Cæsar seem ambitious?

[...]

You all did see that on the Lupercal I thrice presented him a kingly crown, Which hedid thricerefuse: was this ambition?

Yet Brutus says he was ambitious; And, sure, he is an honourable man. I speak not to disprove what Brutus spoke, But here I am to speak what I do know. You all did love him once, not without cause:

What cause withholds you then, to mourn for him?

O judgment! thou art fledo brutish beasts, And men have lost their reason. Bear with me;

My heart is in the coffin there with Cæsar, And I must pause till it come back to me. il prezzo del cui riscatto ha riempito il pubblico tesoro: sembrò questo atto ambizioso in Cesare?

[...]

Tutti vedeste come al Lupercale tre volte gli presentai una corona di re ch'egli tre volte rifiutò: fu questo atto di ambizione?

Eppure Bruto dice ch'egli fu ambizioso; e, invero, Bruto è uomo d'onore.

Non parlo, no, per smentire ciò che Bruto disse, ma qui io sono per dire ciò che io so. Tutti lo amaste una volta, né senza ragione:

qual ragione vi trattiene dunque dal piangerlo?

O senno, tu sei fuggito tra gli animali bruti e gli uomini hanno perduto la ragione.

Scusatemi; il mio cuore giace là nella bara con Cesare e debbo tacere sinché non ritorni da me.

\subsection{Nell'italiano 'non tradotto'}

Si può quindi escludere che l'uso dell'imperfetto che sto illustrando sia tipico della lingua della traduzione o del doppiaggio, per lo meno dall'inglese, anche perché come anticipato lo ritroviamo poi in diverse varietà dell'italiano contemporaneo ed in varî generi testuali o discorsivi, scritti ed orali. Innanzitutto la forma verbale occorre con questo valore nella lingua letteraria, visto che compare ad es. in Pianto antico [1871] di Giosuè Carducci (dalle Rime nuove, Bologna, Zanichelli, 1887), che di séguito riporto integralmente anche per mostrare la contrapposizione istituita dal poeta tra il melograno ancora vivo ed il figlio invece purtroppo ormai morto, e l'isotopia botanica utilizzata per riferirsi a sé ed al figlio avuto in tarda età: «Tu fior della mia pianta / percossa e inaridita, / tu dell'inutil vita / estremo unico fior»:
L'albero a cui tendevi
La pargoletta mano,
Il verde melograno
$\mathrm{Da}$ ' bei vermigli fior
Nel muto orto solingo
Rinverdì tutto or ora,
E giugno lo ristora
Di luce e di calor. 


\begin{abstract}
Tu fior de la mia pianta
Percossa e inaridita,

Tu de l'inutil vita

Estremo unico fior,

Sei ne la terra fredda,

Sei ne la terra negra;

Né il sol più ti rallegra

Né ti risveglia amor.
\end{abstract}

Nella lingua poetica del secondo novecento troviamo quest'uso dell'indicativo imperfetto ad es. in uno dei componimenti di Xenia (in Satura, Milano, Mondadori, 1971), dove Eugenio Montale parla con un sacerdote della particolare religiosità della moglie defunta:

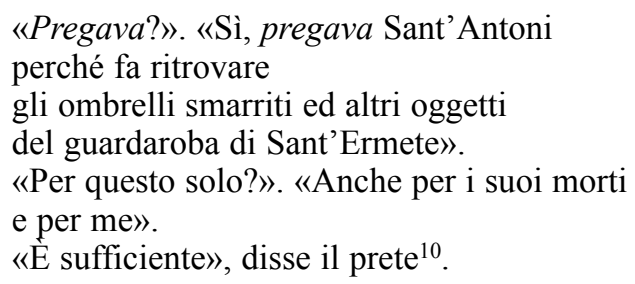

In entrambi i casi siamo di fronte al solito effetto, perché dai versi si capisce che il figlioletto di Carducci non tende più «la pargoletta mano [al] verde melograno», e che Mosca «pregava» ma non prega più...

La stessa forma verbale si trova poi in questo senso in una pellicola italiana contemporanea, Quo vadis, baby? di Gabriele Salvatores (Italia 2004, con fra gli altri Claudia Zanella e Luigi Maria Burruano), in un dialogo tra la protagonista, l'investigatrice privata Giorgia Cantini interpretata da Angela Baraldi, ed il suo amico commissario Bruni impersonato da Andrea Renzi - con l'auto-correzione della parlante dal presente all'imperfetto e la sua motivazione -, e successivamente in una battuta sullo stesso argomento rivolta sempre da Giorgia Cantini ad un altro personaggio, Andrea Berti (Gigio Alberti), professore del suo giovane assistente Lucio (Elio Germano):

GIORGIA: Bruni posso chiederti un favore?

BRUNI: $\quad$ Se posso, volentieri.

Giorgia: $\quad$ Io ho una sorella, Ada... Cioè, avevo una sorella: si è suicidata sedici anni fa.

${ }^{10}$ Eugenio Montale inverte qui un topos dell'agiologia tipica della religiosità popolare italiana, secondo il quale per ritrovare quanto si è perso bisogna pregare Sant'Ermete, che lo cerca frugando nell'armadio di Sant'Antonio - dove appunto vanno a finire gli oggetti smarriti: credo che la cosa possa risultare ancor più interessante ricordando che Sant'Ermete è 'legato' ad Hermes, divinità del pantheon classico patrona fra gli altri anche dei ladri... 


\section{$[\ldots]$}

GIORGIA: $\quad$ Sedici anni fa avevo una sorella, dicono che si sia suicidata.

Ed un'auto-correzione in senso inverso si trova nel frammento seguente, tratto da una intervista al motociclista Loris Capirossi dopo la caduta (e la conseguente frattura esposta a tibia e perone) del collega Valentino Rossi durante il secondo turno delle prove libere del Gran Premio d'Italia sul circuito del Mugello, per il Campionato mondiale categoria Moto GP:

Valentino era... ̀̀ un simbolo [...]. (dal TG 2 delle 13.00 di domenica 6 giugno 2010)

Nell'italiano contemporaneo la forma verbale compare ad es. poi anche nella canzone 4 marzo 1943 di Lucio Dalla e Paola Pallottino (del 1971), usata con riferimento ai genitori dell'autore implicito, come si vede dal testo entrambi morti e qui credo valga la pena di sottolineare lo 'scontro' tra la visualizzazione aspettuale dell'imperfetto e la caratterizzazione azionale del verbo compiere in «Compiva»:

Dice ch'era un bell'uomo e veniva, veniva dal mare, parlava un'altra lingua però sapeva amare;

e quel giorno lui prese mia madre, sopra un bel prato, l'ora più dolce prima d'essere ammazzato.

\section{$[\ldots]$}

Compiva sedici anni quel giorno la mia mamma, le strofe di taverna le cantò a ninna nanna.

E stringendomi al petto che sapeva, sapeva di mare, giocava a far la donna con un bimbo da fasciare.

E forse fu per gioco, o forse per amore che mi volle chiamare come nostro signore. 
Della sua breve vita il ricordo,
il ricordo più grosso,
è tutto in questo nome
che io mi porto addosso.

E nell'italiano parlato comune questo valore funereo dell'indicativo imperfetto può emergere (sia pure come effetto collaterale indesiderato) anche all'interno di scambi dialogici quotidiani come quello 'mimato' nell'esempio successivo:

PARlante A: Dunque, questo tipo era simpaticissimo: era un mio collega di Milano e tutte le studentesse lo amavano alla follia.

PARLANTE B: Ah, è morto? Mi spiace!

PARLANTE A: No no, è ancora vivo, semplicemente non è più mio collega, visto che ora lavoro a Forli!

In quest'ultimo esempio il commento del secondo parlante innesca la mossa di repair del primo, che segnala così che gli imperfetti che aveva utilizzato erano dei semplici passati di sfondo, ${ }^{11}$ senza nessuna implicazione sulla 'fine' (della simpatia) del collega e dell'amore provato per lui dalle sue studentesse...

Ed infine un ultimo esempio reale, per quanto aneddotico, dell'uso dell'imperfetto che sto qui commentando: una giovanissima animalista in nuce, la minore delle due figlie di una coppia di nostri amici di famiglia, aveva trovato un metodo di lotta 'anti-pellicce' assolutamente non aggressivo ma non per questo potenzialmente meno efficace, se non altro a livello dei possibili comportamenti individuali indotti; quando le capitava di incontrare una signora impellicciata l'avvicinava, e guardandola di sotto in su, con un sorriso un po' triste e sbattendo le lunghe ciglia bionde dei suoi dolci occhi azzurri, appena sopra un nasino e due guanciotte cosparsi di lentiggini rossicce, le chiedeva - nell'evidente imbarazzo dei suoi genitori, un docente universitario di filosofia ed un'insegnante di greco e latino del locale liceo classico: «Signora, che animale era?», implicando evidentemente la necessaria morte del precedente 'proprietario naturale' della suddetta pelliccia...

$$
* \quad * \quad *
$$

Se altrove ho tentato in qualche modo di 'potare' con una prospettiva occamistica la selva terminologico-concettuale dei secondo me troppi usi e valori precedentemente riconosciuti all'imperfetto, cercando per lo meno di passare da un lungo elenco ad un sistema organizzato in modo gerarchico, con 'apparentamenti' basati sui diversi valori assunti dalla forma verbale rispetto ai parametri della collocazione temporale, della visualizzazione aspettuale e dell'atteggiamento epistemico del mittente (cfr. Mazzoleni 2009), in questo caso non ho però saputo resistere al furor

${ }^{11}$ Sui concetti di sfondo e primo piano (Background / Foreground) all'interno delle dinamiche narrative, e sui loro due diversi tipi di esponenti formali correlati, cfr Talmy (1975), Hopper (1979ab), Reinhart (1984), Tomlin (1985) e poi Mazzoleni (1991). 
taxonomicus ed alla tentazione nomenclatoria, per cui per l'uso dell'indicativo imperfetto qui sommariamente descritto propongo la quadruplice denominazione che costituisce il titolo di questo mio contributo.

\section{RIFERIMENTI BIBLIOGRAFICI}

HopPer, Paul J. (1979a): «Some Observations on the Typology of Focus and Aspect in Narrative Language», Studies in Language III/1, pp. 37-64.

Hopper, Paul J. (1979b): «Aspect and Foregrounding in Discourse», in T. Givón (ed.), Syntax and Semantics 12: Discourse and Syntax. New York, Academic Press, pp. 213241.

MAZZOLENI, Marco (1991): «Prospettiva funzionale di frase e rilievo informativo nei costrutti ipotattici: due diversi livelli di analisi», Lingua e stile XXVI/2, pp. 151-165.

MAZzoLENI, Marco (1997): «Vocativo, termini di parentela, e vocativo inverso: fra valori di sistema e valori di campo», in L. Agostiniani et alii (eds.), Atti del Terzo Convegno della Società Internazionale di Linguistica e Filologia Italiana (Perugia, 27-29 giugno 1994). Napoli, Edizioni Scientifiche Italiane (Pubblicazioni dell'Università degli Studi di Perugia - Istituto di Linguistica 2), vol. II, pp. 457-475.

MazzolenI, Marco (2009): «Su alcuni usi modali dell'indicativo imperfetto in italiano», relazione invitata al Convegno Internazionale Núcleos. Estudios contrastivos sobre el verbo castellano e italiano / Nuclei. Studi contrastivi sul verbo spagnolo e italiano (Forlì, 26-27 novembre 2009).

ReINHART, Tanya (1984): «Principles of Gestalt Perception in the Temporal Organization of Narrative Text», Linguistics XXII/6, pp. 779-809.

TALmY, Leonard (1975): «Figure and Ground in Complex Sentences», in C. Cogen et alii (eds.), Proceedings of the First Annual Meeting of the Berkeley Linguistics Society (1517 February, 1975), Berkeley (Ca.), BLS, pp. 419-430; ried. ampliata in J. H Greenberg (ed.), Universals of Human Language 4: Syntax. Stanford, Stanford University Press, 1978, pp. 625-649.

TombIN, Russell S. (1985): «Foreground-Background and the Syntax of Subordination», Text V/1-2, pp. 85-122. 